\title{
Contribuições da produção de autoconsumo em quintais para a segurança alimentar e nutricional e renda em Jangada, Baixada Cuiabana, MT
}

\author{
Contributions of self-consumption production in homegardens for food and nutritional security \\ and income Jangada, Baixada Cuiabana, MT
}

\author{
Cleomara Nunes do Amaral ${ }^{1}$ \\ Gabriela Peixoto Coelho-de-Souza ${ }^{2}$ \\ Ilaine Schuch $^{3}$ \\ Marcelino de Souza ${ }^{4}$
}

\begin{abstract}
Resumo
O objetivo principal deste trabalho é apresentar os resultados de pesquisa sobre a contribuição dos quintais na segurança alimentar e nutricional e nas rendas das famílias de agricultoras tradicionais. Para tal, foi constituída uma amostra aleatória proporcional ao tamanho da população e foram pesquisadas 90 famílias em 7 comunidades rurais do município de Jangada, Baixada Cuiabana, estado do Mato Grosso. Os dados foram coletados no período de agosto de 2012 a julho de 2013. Para a análise de consumo alimentar e aspectos de segurança alimentar das famílias, os dados foram coletados por meio da técnica de inquérito de consumo alimentar familiar. Mensurou-se o valor econômico do autoconsumo das famílias traduzido sob a forma de renda, considerando-se os preços praticados ao consumidor. A produção obtida oferece nutrientes importantes, ficando ausentes os alimentos mais ricos em minerais, os quais são produzidos em menor quantidade pelas famílias. A produção dos quintais parece não ser suficiente para garantir a segurança alimentar das famílias, especialmente no que se refere ao consumo de alguns macronutrientes e vitaminas em níveis adequados. No entanto, o autoconsumo, que muitas vezes se apresenta como uma renda invisível, contribui economicamente para a manutenção das famílias, bem como para garantir ao menos parcialmente a segurança alimentar dos agricultores e de suas famílias.
\end{abstract}

Palavras-chave: Quintais. Agricultura tradicional. Segurança alimentar. Multifuncionalidade.

\footnotetext{
Abstract

The main objective of this work is to present the results of research on the contribution of homegardens in food and nutrition security and the incomes of traditional farmers families. To this

${ }^{1}$ Professora da Secretaria de Educação do Estado do Mato Grosso, E-mail: cleomara.cefapro@gmail.com.

2 Professora da Faculdade de Ciências Econômicas e do Programa de Pós Graduação em Desenvolvimento Rural da Universidade Federal do Rio Grande do Sul. E-mail: gabrielacoelhodesouza@yahoo.com.br.

${ }^{3}$ Professora Adjunta do Departamento de Nutrição/Faculdade de Medicina da Universidade Federal do Rio Grande do Sul. E-mail: ischuch@hcpa.edu.br.

${ }^{4}$ Professor Associado da Faculdade de Ciências Econômicas e do Programa de Pós Graduação em Desenvolvimento Rural da Universidade Federal do Rio Grande do Sul. E-mail: marcelino.souza@uol.com.br.
} 
end, it established a proportional random sample of the size of the population and was surveyed 90 families in seven rural communities in the municipality of Jangada, Baixada Cuiabana, state of Mato Grosso. Data were collected from August 2012 to July 2013. For the analysis of food consumption and aspects of household food security data were collected through the household food consumption survey technique. Measured to the economic value of own consumption of families, translated in the form of income considering the prices charged to consumers. The production obtained provides important nutrients, getting away the foods richest in minerals, which are produced in smaller quantities by families. The production of homegardens seems not to be sufficient to ensure food safety of the families, particularly as regards the consumption of some macronutrients and vitamins in adequate levels. However, the self-consumption, which often presents itself as an invisible income economically contributes to the maintenance of the families, and to ensure at least partially the food security of farmers and their families.

Keywords: Homegardens. Traditional agriculture. Food security. Multifunctionality.

\section{Introdução}

De acordo com Freitas e Pena (2007), a Segurança Alimentar e Nutricional (SAN) significa a permanência de acesso ao alimento de qualidade, não apenas quanto à disponibilidade do alimento, mas, sobretudo, em relação à segurança que o indivíduo/família e o coletivo têm com o alimento, construída na complexidade das relações sociais.

Segundo a FAO (Organização das Nações Unidas para Alimentação e Agricultura, 2014) a função da Segurança Alimentar está relacionada à quantidade e qualidade dos alimentos adquiridos pelas famílias, de modo a satisfazer as necessidades diárias, tendo em vista a obtenção de um padrão de vida ativa e saudável. Ao abordar o tema da SAN, vários aspectos precisam ser analisados. Segundo a FAO (2014), há 4 dimensões reconhecidas internacionalmente em estudos de SAN: disponibilidade, acesso, utilização dos nutrientes e estabilidade. De acordo com Pieroni e Price (2006), o conhecimento tradicional contribui para a melhora do estado nutricional e saúde humana. Atualmente, a promoção da segurança alimentar é vista como a principal função da agricultura para a sociedade, considerada um bem público, junto com a biodiversidade, paisagem natural e herança cultural. Maluf (2003) destaca que a partir do enfoque da multifuncionalidade, poderia se garantir e incentivar o autoconsumo das famílias rurais e a segurança alimentar, os quais são cruciais para a dinamização social e econômica do espaço rural brasileiro.

Assim, o enfoque da multifuncionalidade não surge para diminuir a importância da agricultura, mas para ressaltar novas e antigas funções, valorizar suas múltiplas contribuições e 
aptidões, que vão além da atividade agrícola, e que estão sendo entendidas, absorvidas e implementadas pelos agricultores, cada vez mais sensíveis às transformações do meio rural, procurando se adaptar à nova realidade. Carneiro e Maluf (2003) ao aplicarem, como referencial analítico, a noção de multifuncionalidade no contexto brasileiro, identificam quatro funções chave da agricultura: a. Reprodução socioeconômica das famílias rurais; b. Manutenção do tecido social e cultural; c. Promoção da segurança alimentar das próprias famílias rurais e da sociedade; d. Conservação da agrobiodiversidade e manutenção da paisagem rural.

Nesse sentido, neste artigo trataremos de abordar a função dos quintais ${ }^{5}$ na segurança alimentar das famílias, abrangendo especialmente a produção para o autoconsumo, mais especificamente a produção de plantas alimentícias, frutíferas, hortaliças e plantas medicinais, que podem ser usadas no cuidado da saúde da família e da comunidade. Nascimento (2003) destaca a importância dos quintais domésticos e da agrobiodiversidade em relação à alimentação e renda familiar, ressaltando sua relevância para a diversificação dos alimentos presentes no consumo diário.

Estudos de Nascimento (2003) com relação à segurança alimentar, à quantidade de consumo e frescor dos alimentos apontam que crianças pobres pertencentes às famílias de agricultores possuem um diferencial nutricional superior à de crianças pobres de famílias de não agricultores. Assim, a ausência do quintal seria um fator de restrição da dieta alimentar, especialmente de alimentos que são fontes de vitaminas, minerais e fibras, como por exemplo, as hortaliças e frutas.

Para Pasa (2004), a produção nos quintais permite à população manter uma alimentação mais saudável, reduz a dependência de produtos adquiridos externamente, ocasiona impactos mínimos sobre o ambiente, conserva os recursos vegetais e a riqueza cultural, fundamentada no saber e na cultura dos moradores locais. Considerando o aspecto cultural, mais especialmente a relação de gênero, é possível notar também que a divisão de trabalho está muito presente nos espaços rurais, especialmente nos espaços destinados para aprovisionamento do autoconsumo da família, sendo este de responsabilidade predominantemente feminina (MENASCHE et al., 2008), outro aspecto importante relacionado ao desenvolvimento rural.

\footnotetext{
${ }^{5}$ Para Kumar e Nair (2004), os quintais ou homegardens possuem longa tradição em países tropicais. Representam uma das formas mais antigas de manejo da terra, e que através da produção de múltiplas espécies são responsáveis por prover e sustentar milhões de pessoas economicamente. Segundo os mesmos autores, apesar de ser uma prática antiga, os quintais são menos estudados do que deveriam, em razão de sua complexidade e importância.
} 
De acordo com Wedig (2009), em estudo realizado no estado do Rio Grande do Sul, as atividades desempenhadas pelas mulheres são geralmente voltadas para atender o consumo das famílias. Zanetti e Menashe (2007) também apontam que os alimentos proporcionados pelas mulheres são considerados "miudezas", enquanto aqueles produzidos pelos homens nas roças, lavouras ou outros espaços são considerados de maior valor, isso porque são destinados ao comércio. Na região Centro-Oeste do Brasil, especialmente no estado de Mato Grosso (PASA, 2004; AMARAL, GUARIM NETO, 2008; MOREIRA, GUARIM NETO, 2009) essa situação não é diferente. A maior parte do trabalho realizado pelas mulheres na agricultura é desempenhada em áreas próximas à residência, como o quintal, destinado ao trato de animais de pequeno porte como porcos e galinhas, o cultivo de frutas, verduras, legumes e a manutenção de outras plantas voltadas especialmente para o consumo familiar.

Zanetti e Menashe (2007) afirmam que a produção de alimentos voltada para consumo das famílias rurais desempenha importante papel como estratégia de reprodução social. No entanto, como essa produção se apresenta à margem das relações de mercado, e é realizada principalmente por mulheres, ela é pouco valorizada. As mulheres são responsáveis pela saúde da família, são as guardiãs dos saberes locais e dos conhecimentos úteis para a lida na roça, cozinha, criações e horta, que vincula a culinária à agricultura (RAMOS, 2007). Na horta, junto aos vegetais utilizados na salada, condimentos e temperos, as mulheres mantêm com frequência algumas ervas para a preparação de chás e sucos, utilizados em casos de necessidade de pessoas da família ou próximas. Vale destacar que na maioria dos casos, essas comunidades estão situadas em locais muito distantes da cidade e de postos médicos, utilizando as plantas medicinais produzidas em seus quintais ou em outros espaços próximos, matas do entorno e terrenos vazios, como primeira alternativa.

Menashe et al. (2008) destacam ainda que a falta de valorização do trabalho feminino está entre os motivos de seu distanciamento do trabalho agrícola, o que contribui para a tendência de redução da produção de alimentos voltada ao autoconsumo. Por conseguinte, o não reconhecimento do trabalho realizado pelas mulheres agricultoras concorreria para uma situação de insegurança alimentar.

Ramos (2007) destaca as transformações nos hábitos alimentares e preferências que refletem na produção e na mesa dos agricultores. Alguns produtos, antes amplamente cultivados nos 
quintais, vêm atualmente perdendo espaço na mesa de agricultores tradicionais ${ }^{6}$, como por exemplo, as raízes e os tubérculos. De acordo com estudo realizado por Ramos em Maquiné, Rio Grande do Sul, as raízes e tubérculos são alimentos bastante apreciados, podendo ser consumidos em forma de assados ou cozidos e, ainda, utilizados especialmente pela população mais antiga, na preparação de massas, como por exemplo, pão com aipim, batata-doce, cará ou inhame, o que garantia um enriquecimento nutricional do pão consumido. Hoje em dia, muitos desses produtos caseiros, como pães e massas, estão sendo substituídos por produtos altamente processados.

Em Mato Grosso não há pesquisas desse tipo que permitam comprovar essa substituição, mas há um visível aumento no consumo de produtos altamente processados, até mesmo pela maior facilidade de acesso em detrimento do consumo e produção de alimentos caseiros.

Assim, percebe-se que a agrobiodiversidade mantida em espaços próximos às residências, como os quintais, apesar das transformações ocorridas ao longo dos últimos anos, de uma forma geral, são valiosas fontes de alimentos e nutrientes para as famílias rurais, além de se apresentar como alternativa econômica para o consumo de alimentos necessários e saudáveis, proporcionando às famílias, entre outros benefícios, segurança alimentar e nutricional, e melhor qualidade de vida (AMARAL; GUARIM NETO, 2008).

O quintal das agricultoras tradicionais de Jangada, região da Baixada Cuiabana - MT, está intimamente associado à cultura e tradição cuiabana, sendo um espaço de manejo, especialmente da mulher, que desempenha um papel central nos modos de vida dessas famílias e um importante papel relacionado à alimentação e segurança alimentar das famílias. A função dos quintais na segurança alimentar das famílias abrange especialmente a produção para o autoconsumo, mais especificamente a produção de plantas alimentícias, frutíferas, hortaliças e plantas medicinais, que podem ser usadas no cuidado da saúde da família, da comunidade e das pessoas do entorno. Nascimento (2003) destaca a importância dos quintais domésticos e da agrobiodiversidade em relação à alimentação, bem como à renda familiar, ressaltando sua relevância para a diversificação da produção de alimentos presentes no consumo diário das famílias.

Esta pesquisa reconhece a complexidade e a importância do tema, entretanto, por questões metodológicas, limitou-se a abordar alguns aspectos referentes à disponibilidade e acesso dos

\footnotetext{
${ }^{6}$ Mattos et al. (2010, p. 228), citando Schneider (2006) e Abramovay (1998), afirmam que "os agricultores tradicionais podem ser entendidos como sociedades parciais com uma cultura parcial, integrados de modo incompleto a mercados imperfeitos, que representam um modo de vida caracterizado pela personalização dos vínculos sociais e pela ausência de contabilidade formal nas operações produtivas".
} 
alimentos, especialmente quanto à produção dos quintais e análise nutricional das famílias. Sendo assim, o objetivo da pesquisa foi de identificar e analisar a contribuição da produção de autoconsumo de agricultores tradicionais em quintais, especificamente em relação à segurança alimentar e nutricional e também de renda no contexto do município de Jangada, Baixada Cuiabana, estado do Mato Grosso.

\section{Procedimentos metodológicos}

O presente estudo foi desenvolvido no município de Jangada, Território da Baixada Cuiabana, estado de Mato Grosso. A região se destaca pela presença de agricultores tradicionais inscritas em uma mesma identidade cultural em função de fatores históricos relacionados à origem de Mato Grosso e onde a presença dos quintais é muito relevante. Todos os municípios rurais do território apresentam grande parte de sua população dependente de benefícios sociais do governo. O índice de dependência médio dos municípios da Baixada Cuiabana é maior do que a média do Estado (54,9\%). A escolha do município de Jangada, como local de pesquisa, seguiu alguns critérios, tais como: está localizado em uma área de ocupação tradicional do estado, denominado Território da Baixada Cuiabana; possui maior parte da população concentrada em áreas rurais; a economia local é voltada à agricultura, especialmente para o autoconsumo e criação de gado; grande número de pequenos agricutores ou agricultoras; o município apresenta baixo Índice de Desevolvimento Humano (IDH), com alto índice de dependência de recursos governamentais; é considerado um dos municípios mais pobres do estado, recebendo por isso atenção prioritária em programas e ações que visem ao combate e erradicação da pobreza.

Nesse contexto, o município de Jangada se destaca, tendo mais de $75 \%$ da população em situação de dependência. Esse fato demonstra que nos municípios do território como um todo, e em especial em Jangada, existe uma quantidade grande de moradores que dependem de recursos externos para a sua manutenção (GARBIN et al., 2006).

A constituição da amostra desta pesquisa partiu de um cadastro de agricultores(as) com um número total de 797 famílias e 28 comunidades. Assim, enquadraram-se nas características mencionadas as seguintes comunidades: Cascavel, Mato Grosso, Mutum, Quilombo, Raizama, Ribeirão das Pedras e Vaquejador. A partir do reconhecimento prévio da população que seria 
estudada nesta pesquisa, realizou-se, em um primeiro estágio, uma amostra aleatória proporcional ao tamanho da população para definir o número de famílias que seriam entrevistadas que permitisse uma representação segura da realidade das comunidades.

Partiu-se do pressuposto de que a variância entre as comunidades que compõem o grupo é semelhante e que o consumo, mesmo apresentando uma diversidade, teria um comportamento razoavelmente homogêneo nas comunidades. Sendo assim, a amostra obtida abrangeu acima de $25 \%$ das famílias e seus respectivos quintais. Foram entrevistadas 90 famílias em 7 comunidades. Os dados foram coletados pela pesquisadora no período de agosto de 2012 a julho de 2013. Foram realizadas visitas periódicas semanais às comunidades rurais de Jangada durante esse período, sendo que cada família que compôs a amostra foi entrevistada apenas uma vez. Aos agricultores(as) participantes foi lido e apresentado o Termo de Consentimento, contendo informações sobre a importância da pesquisa e outras questões relevantes sobre a pesquisa e o pesquisador. As entrevistas foram realizadas obedecendo aos princípios éticos, e os(as) agricultores(as) foram entrevistados(as) depois de informados(as) do objetivo da pesquisa e do consentimento prévio.

Vários aspectos foram levantados na coleta de dados da pesquisa, mas para a análise do consumo alimentar e aspectos de segurança alimentar das famílias os dados foram coletados por meio da técnica de inquérito de consumo alimentar familiar, desenvolvida por Galeazzi et al. (1996), já que a técnica é tradicional no meio acadêmico e familiar aos autores(as). Ela consistiu no levantamento sobre os itens consumidos pelas famílias no mês anterior ao da entrevista realizada através de questionário de frequência de consumo alimentar (QFCA). Dessa forma, especificamente para a coleta dos dados de consumo de alimentos, a lista utilizada foi composta de 80 alimentos distribuídos em 11 grupos. O peso e/ou quantidade consumida por cada família foi a quantidade aproximada informada pelo entrevistado. Inicialmente, os dados relativos ao consumo de alimentos foram digitados em planilhas do programa Excel, versão 6.0, sendo avaliada a consistência, por meio da realização de alguns cruzamentos iniciais, para a identificação de possíveis erros. A partir das planilhas sobre as quantidades dos alimentos consumidos por família/mês foi realizada a conversão dessas quantidades para unidade única de quilograma $(\mathrm{Kg})$ ou litros $(\mathrm{L})$.

Os pesos líquidos dos alimentos foram importados para o programa SPSS (Statistical Product and Service Solutions), versão 18.0, para análise da composição química dos alimentos consumidos pelas famílias pesquisadas. As quantidades de cada alimento que cada família declarou ter consumido no mês anterior foram, então, analisadas quanto a sua composição e os resultados das 
quantidades de cada nutriente foram divididos pelo número de dias do mês para obter a média diária para cada nutriente em cada uma das famílias pesquisadas. A análise dos alimentos foi realizada utilizando-se a Tabela Brasileira de Composição dos Alimentos (TACO; NEPA-UNICAMP, 2011). Foram determinados os valores para calorias a partir dos macronutrientes proteínas, carboidratos e lipídios. Os micronutrientes e vitaminas analisados foram: cálcio $(\mathrm{mg})$, ferro $(\mathrm{mg})$, vitamina A $(\mu \mathrm{g})$ e vitamina C (mg). Também foram analisadas as quantidades de fibras. As necessidades nutricionais médias para cada família foram calculadas a partir das recomendações contidas nas Dietary Reference Intakes (DRI, 2001), considerando a idade e o sexo de cada indivíduo da família. $\mathrm{O}$ consumo de calorias e micronutrientes foi considerado adequado quando este atingia uma faixa de valores entre 90 e $110 \%$ em relação aos valores médios recomendados. Para encontrar os percentuais de adequação de calorias e de micronutrientes, compararam-se os dados do consumo médio da família/dia com as necessidades médias da família/dia.

A partir da totalidade dos alimentos consumidos e que são produzidos nos quintais, avaliouse, comparativamente, a contribuição dos quintais para o autoconsumo das famílias. Para medir o valor econômico do autoconsumo das famílias, traduzido sob a forma de renda, adotou-se a metodologia proposta por Garcia Jr. (1989), considerando os preços praticados ao consumidor.

\section{Resultados e discussão}

\section{Aspectos nutricionais e de segurança alimentar das famílias}

Para as agricultoras tradicionais de Jangada, a segurança alimentar das famílias está intimamente relacionada à qualidade da alimentação. Além disso, o manejo dos quintais e os cuidados relacionados à alimentação das famílias é uma atividade quase sempre ligada às mulheres, e em $92 \%$ das entrevistas realizadas as mulheres foram indicadas como principais informantes da casa e responsáveis. Por isso, doravante na apresentação e análise dos dados denominaremos genericamente agricultoras entrevistadas.

A preocupação em consumir alimentos livres de agrotóxicos, utilizando adubos orgânicos, foi constante nos depoimentos das agricultoras. Ao serem questionadas, $85 \%$ das famílias acreditam possuir uma alimentação adequada. No campo da cultura, o sentido do termo segurança, em analogia ao acesso aos alimentos, é expresso pelos sujeitos em seu mundo cotidiano (FREITAS; 
PENA, 2007). Vale ressaltar que, segundo as informantes da pesquisa, o restante dos alimentos produzidos pode ainda ser utilizado na alimentação dos animais e como adubo para as plantações.

Entre os principais grupos de alimentos consumidos estão primeiramente o grupo dos cereais, seguido do grupo das raízes e tubérculos. A produção dos quintais no primeiro grupo (cereais) é baixa; já no segundo grupo é importante, suprindo em mais de $50 \%$ as necessidades da família, com destaque para a produção da mandioca, farinha e batata-doce. Em seguida, por ordem de consumo, aparece o grupo das carnes, seguido do grupo do leite e derivados. Em 90\% dos quintais registrou-se a produção de galinhas, criadas no sistema caipira, livres nos quintais. Não obstante, a produção de galinhas nos quintais representa $72 \%$ do consumido e $62 \%$ de ovos.

Entre os grupos de alimentos consumidos em menor quantidade, está o grupo das verduras e legumes, seguido do grupo das frutas. Apesar de apresentar um consumo menor que nos demais grupos de alimentos, a importância do quintal aqui é marcante, pois a maior parte do alimento consumido é também produzida no quintal (Gráfico 1).

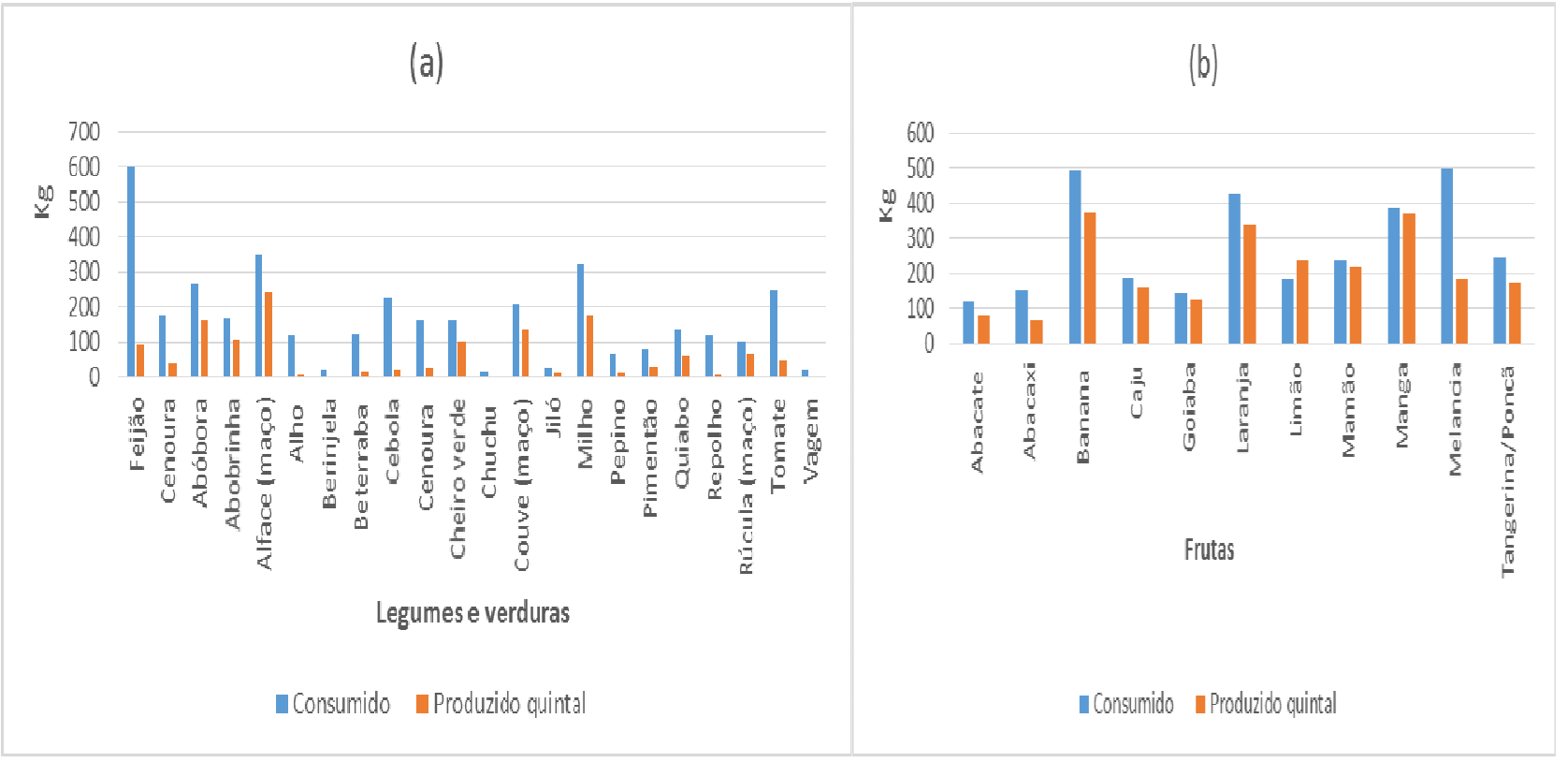

GRÁFICO 1 - RELAÇÃO MENSAL ENTRE A QUANTIDADE PRODUZIDA E CONSUMIDA NO GRUPO DOS LEGUMES E VERDURAS (A), SEGUIDA DO GRUPO DAS FRUTAS (B).

FONTE: elaboração própria (2014) a partir de dados coletados em pesquisas de campo (2012-2013).

Entre os principais produtos beneficiados, $36 \%$ das famílias informaram que beneficiam a farinha de mandioca nos quintais. Apenas $7 \%$ das famílias beneficiam mais de 10 produtos. Percebe-se que os quintais jangadenses representam para as agricultoras, assim como o proposto por 
Niñez (1984), uma estratégia adaptativa na economia de famílias com baixo poder aquisitivo, ao utilizarem como parte da alimentação familiar etnoespécie, geralmente, frutíferas cultivadas nos quintais ou mesmo espécies nativas locais. Além disso, Niñez (1984) e Santos (2004) destacam a possibilidade de complementação da renda familiar com a venda de alguns vegetais produzidos nos quintais. Nesse caso, as hortaliças e animais, como as galinhas e porcos, são produtos agropecuários dos quintais que além de consumidos são comercializados complementarmente.

Conforme foi exposto na seção metodológica do artigo, a análise dos aspectos nutricionais das famílias foi realizada a partir aplicação do questionário de frequência de consumo alimentar QFCA. A partir deste, estimou-se o aporte de energia e nutrientes de acordo com a idade e sexo da pessoa, a partir da comparação com as recomendações da Organização Mundial da Saúde (OMS). A análise do consumo de calorias e de macro e micronutrientes das famílias agricultoras de Jangada demonstra que, de modo geral, as famílias $(77,8 \%)$ possuem um consumo maior que o recomendado (Tabela 1 ).

TABELA 1 - DISTRIBUIÇÃO DAS FAMÍLIAS DE AGRICULTORAS TRADICIONAIS DE JANGADA, MT, DE ACORDO COM OS PERCENTUAIS DE ADEQUAÇÃO DE CONSUMO DE CALORIAS

\begin{tabular}{cc}
\hline Pontos de corte das recomendações & $\mathbf{N}(\%)$ \\
\hline$<80 \%$ & $9(10)$ \\
$80 \%-110 \%$ & $11(12,2)$ \\
$>110 \%$ & $70(77,8)$ \\
\hline
\end{tabular}

\section{Total} 90

FONTE: elaboração própria a partir de dados coletados em pesquisas de campo (2014).

O consumo de proteínas está no limite, com uma tendência a elevado, e o consumo de gorduras das famílias está acima da faixa de recomendação para uma alimentação equilibrada (Tabela 2). A alta ingestão de carne, a introdução de alimentos altamente processados na dieta, bem como alguns hábitos alimentares locais, como a utilização de gordura de origem animal na preparação de alimentos, contribui para a elevação do consumo de gordura acima do ideal. 
TABELA 2 - PROPORÇÃO MÉDIA DE MACRONUTRIENTES NA COMPOSIÇÃO DA ALIMENTAÇÃO DAS FAMÍLIAS DE AGRICULTORAS TRADICIONAIS DE JANGADA, MT

\begin{tabular}{ccc}
\hline Macronutrientes & Média (DP) & Recomendações (\%) \\
\hline Proteínas & $12,6 \pm 2,3$ & $10 \%-15 \%$ \\
Carboidratos & $48,0 \pm 7,9$ & $60 \%-70 \%$ \\
Lipídios & $38,6 \pm 6,8$ & $20 \%-30 \%$ \\
\hline
\end{tabular}

FONTE: elaboração própria a partir de dados coletados em pesquisas de campo (2014).

O consumo de calorias em excesso, conforme apresentado na Tabela 1, pode ser considerado como um indicativo para investigação de doenças crônicas na população em questão. Da mesma forma, o consumo em excesso de gordura entre as famílias estudadas também pode estar associado ao fator cultural. Assim como abordado por Woortmann (2007) em estudos de famílias camponesas, a comida ideal, no sistema antigo, era aquela rica em gordura. De acordo com a autora, a gordura é apontada como uma comida forte, dentro de uma classificação da comida ligada ao trabalho. A comida forte fornece a energia necessária para a realização do trabalho pesado, como é o realizado na roça (WEDIG, 2009). Todavia, com a tendência ao sedentarismo da população, seja no meio urbano ou rural, essa questão passa a ser importante, pois se relaciona ao sobrepeso e à obesidade não apenas entre a população adulta, mas também entre jovens e crianças em curto e médio prazo.

$\mathrm{Na}$ Tabela 3 apresentam-se os dados referentes ao consumo de vitaminas e micronutrientes, demonstrando que a maioria das famílias possui consumo inadequado (abaixo do recomendado) de cálcio e vitamina A. À primeira vista, a análise nutricional das famílias pode apresentar indicativo de uma alimentação de baixo valor nutricional.

As principais fontes de cálcio são o leite e seus derivados, como o iogurte e o queijo, mas os micronutrientes também estão presentes em algumas frutas e em vegetais de cor escura, como na laranja, salsinha, rúcula, couve, feijão, espinafre, quiabo e brócolis. Apesar do leite se constituir no alimento consumido por todas as 90 famílias entrevistadas, ele é produzido apenas por 22 famílias e, mesmo assim, é um alimento consumido quase exclusivamente por crianças e adolescentes. Pessoas com osteoporose ou histórico da doença na família devem ter uma alimentação rica em cálcio, assim como mulheres na fase da menopausa e crianças, para prevenir problemas relacionados à alteração hormonal. 
TABELA 3 - DISTRIBUIÇÃO DAS FAMÍLIAS DE AGRICULTORAS TRADICIONAIS DE JANGADA, MT, DE ACORDO COM O CONSUMO DE MICRONUTRIENTES

\begin{tabular}{lccc}
\hline \multirow{2}{*}{ Micronutriente } & \multicolumn{3}{c}{ Percentuais de adequação } \\
\cline { 2 - 4 } & $<90 \%$ & $90 \%-110 \%$ & $>110 \%$ \\
\hline Cálcio & $\mathbf{8 0}(\mathbf{8 8 , 9 )}$ & $\mathbf{4 ( 4 , 4 )}(\mathbf{6 , 7 )}$ \\
Ferro & $11(12,2)$ & $4(4,4)$ & $75(83,3)$ \\
Vitamina A & $\mathbf{7 0 ( 7 7 , 8 )}$ & $\mathbf{4 ( 4 , 4 )}$ & $\mathbf{1 6}(\mathbf{1 7 , 8 )}$ \\
Vitamina C & $25(27,8)$ & $5(5,6)$ & $60(66,7)$ \\
Fibra & $15(16,7)$ & $8(8,9)$ & $67(74,4)$ \\
\hline
\end{tabular}

FONTE: elaboração própria a partir de dados coletados em pesquisas de campo (2014).

A vitamina A pode ser encontrada em alimentos de origem animal como o fígado, o rim, o leite integral, os queijos, a manteiga e os ovos. Alguns alimentos de origem vegetal ricos em betacaroteno, que serão convertidos em vitamina A, são: couve-manteiga, folhas de beterraba, folhas de cenoura, rúcula, abacate, caqui, manga e acerola. O consumo abaixo do ideal de vitamina A, assim como o cálcio, pode estar relacionado ao baixo consumo de alimentos como o leite e os seus derivados. Já com relação aos vegetais ricos em vitamina A e que são produzidos nos quintais, estes se restringem basicamente à couve e à rúcula. Mesmo assim, essa produção ocorre apenas em aproximadamente $30 \%$ das famílias.

Nota-se que há a produção de uma variedade de alimentos que poderia suprir a demanda dessa população por boa parte das vitaminas e sais minerais na quantidade recomendada, mas é preciso haver uma política de informação e qualificação para que ocorra o aprimoramento da produção das famílias, garantindo que os alimentos sejam consumidos em qualidade e quantidade necessárias, durante o ano todo, para que se tenha uma condição de segurança alimentar das famílias. O consumo alimentar dessas famílias está intimamente associado aos alimentos que elas produzem e à economia proporcionada pela produção destes.

Além do fator produtivo, o consumo e a alimentação das famílias rurais estão também relacionados aos elementos social e cultural. Adicionalmente, há que se destacar que ainda que sejam comunidades rurais, estas podem sofrer os efeitos da difusão da urbanização e da globalização sobre os hábitos alimentares, com a inserção de produtos altamente processados nas dietas das famílias. 
A partir da lista mensal dos alimentos consumidos pelas famílias do município de Jangada, é possível identificar que, de um total de 80 produtos consumidos pelas famílias durante o mês, 20 deles são produzidos nos quintais das famílias, representando $25 \%$ dos alimentos consumidos. Independentemente do valor ou porcentagem real do que foi economizado com a produção dos quintais, é fato que a produção deles aumenta o poder de compra, uma vez que as pessoas deixam de adquirir produtos externos, fortalecendo a economia local. No caso das comunidades tradicionais de Jangada, o método levou em consideração apenas os alimentos produzidos nos quintais e que são usados exclusivamente para alimentação da família. Entre as 90 famílias de agricultoras que fizeram parte da amostra da pesquisa, 20 famílias comercializam a produção excedente dos quintais (representando $22 \%$ ), o que nos parece muito relevante.

A contribuição dos quintais manifesta-se, também na presença de um grande número de variedades de determinadas espécies (EICHEMBERG; AMOROZO; MOURA, 2009). Esse é o caso da manga, da banana e da mandioca nos quintais de Jangada.

\section{Os quintais, a segurança alimentar e a renda}

Considerando apenas os resultados da análise nutricional das famílias, poderia se concluir que o consumo alimentar familiar não satisfaz a característica de uma alimentação adequada em termos nutricionais. No entanto, através da lista de espécies produzidas nos quintais, nota-se que há o cultivo de alimentos que contêm esses nutrientes e poderiam suprir a demanda das famílias na quantidade recomendada. Conforme mencionado, há, portanto, que se aprimorar a forma de utilização dos nutrientes através do conhecimento, permitindo a realização de escolhas e, sobretudo investimento na educação alimentar.

Com relação ao beneficiamento, $28 \%$ das famílias não beneficiam produtos nos quintais. $\mathrm{O}$ beneficiamento da farinha de mandioca é feito por $36 \%$ das famílias, seja nos quintais, de modo tradicional, ou em farinheiras comunitárias, principalmente para o autoconsumo da família. Entretanto, $80 \%$ das agricultoras já produziram ou conhecem as técnicas de produção tradicional da farinha de mandioca e do polvilho, evidenciando uma diminuição da atividade. Essa tradição mantém-se hoje em dia, principalmente, na memória das agricultoras mais antigas.

Em $70 \%$ das famílias a renda proveniente da produção familiar representa menos de $50 \%$ da renda total familiar. Apenas $30 \%$ das famílias têm como renda principal a comercialização de produtos da agricultura. A renda média mensal das famílias foi de aproximadamente R\$1.139,00. 
Nesse cálculo não está incluída a renda proveniente do autoconsumo dos quintais, calculada em aproximadamente $\mathrm{R} \$ 363,52$. Na medida em que se inclui esse valor, a renda familiar média mensal sobe para R \$ 1.502,52. Nesse contexto, o valor do autoconsumo equivale a $24,2 \%$ da renda.

Ao traçar um paralelo com os dados evidenciados por IPEA (2013), de que a renda média do autoconsumo no estado de Pernambuco, a mais baixa do país, é de R $\$ 1.449,32$, representando $7,17 \%$ da proporção entre o valor total do autoconsumo e o valor da produção total, é possível concluir que as agricultoras de Jangada têm uma grande dependência da produção do quintal, em que o autoconsumo, apenas do quintal, representa $24,2 \%$ da renda, valor muito maior do que aquele mencionado no trabalho citado anteriormente.

Um aspecto a destacar nesta pesquisa é que a região também vive constantes processos de transformação nos modos de vida das agricultoras, dado que parte dos mais jovens está buscando trabalho nas cidades, ocorrendo diminuição das áreas de agricultura e a perda da agrobiodiversidade associada às etnoespécies cultivadas, principalmente nas áreas de roça. Embora as práticas e conhecimentos sobre o manejo da agrobiodiversidade e o trabalho no quintal estejam em processo de transformação, ficando retidos apenas na memória da geração atual, ressalta-se a importância dos quintais para o fortalecimento do sistema cultural, muito presente na comunidade.

O fortalecimento da agricultura tradicional no âmbito das políticas em segurança alimentar e nutricional se centra na inclusão da produção em novos mercados, valorização da cultura, em especial a alimentar, crédito, incentivo ao autoconsumo, promoção dos quintais, produção de alimentos saudáveis, com qualidade e quantidades necessárias, garantindo a segurança alimentar e nutricional das famílias, além de favorecer a manutenção da agrobiodiversidade e os modos de vida tradicionais. Além disso, como parte do Direito Humano à Alimentação Adequada, o acesso aos meios para que as famílias possam ser sujeitas de direitos é dever do Estado. É nesse contexto que a segurança alimentar e nutricional, ao considerar o respeito à diversidade étnica e cultural da população, é também um potente instrumento nessa construção. As ações/estratégias em segurança alimentar e nutricional são basilares às de desenvolvimento territorial, atendendo aos princípios de sustentabilidade e proporcionando a multifuncionalidade da agricultura familiar.

Os instrumentos destacados anteriormente permitem articular dimensões éticas, políticas e científicas em escalas locais a internacionais, constituindo-se em mecanismos de conservação, justiça e promoção. Pode-se lançar mão destes, em conjunto ou separadamente, para o fortalecimento dos modos de vida das agricultoras tradicionais que vivem em áreas de Cerrado, 
associados à agrobiodiversidade domesticada e nativa, a qual integra seus modos de vida e sua segurança alimentar.

\section{Considerações finais}

Entre muitos autores, o quintal se apresenta com uma importante função de segurança alimentar, ao abordar a questão da multifuncionalidade da agricultura familiar e, particularmente nesta pesquisa, esse aspecto se tornou também evidente.

A contribuição dos quintais para a segurança alimentar e nutricional pode ser verificada nas análises dos resultados sobre o consumo dos alimentos das famílias, o qual revelou que a preocupação atual não se encontra no consumo de hortaliças, legumes e frutas, pois graças à presença dos quintais, as famílias mostram-se regularmente supridas dos nutrientes existentes nesses alimentos. Na verdade, para as famílias que detêm quintais, faltam os alimentos mais ricos em minerais, como cálcio e ferro, que são encontrados no leite e seus derivados e nas carnes, respectivamente. Esses alimentos não são produzidos em quantidades suficientes pelas famílias para o abastecimento; por isso, geralmente devem ser adquiridos, de forma complementar, no comércio, por preços às vezes pouco acessíveis quando os produtos se encontram disponíveis.

Apesar de algumas deficiências, o fato é que a produção para autoconsumo nos quintais representa para algumas agricultoras a única e principal fonte de frutas, verduras e legumes que consomem. Caso contrário, a ingestão de importantes nutrientes estaria comprometida, prejudicando seriamente a saúde e segurança alimentar de suas famílias.

Nesse sentido, o fortalecimento da agricultura tradicional no âmbito das políticas públicas de segurança alimentar e nutricional poderia se centrar na inclusão da produção em novos mercados, valorização da cultura, em especial a alimentar, crédito, incentivo ao autoconsumo, promoção dos quintais, produção de alimentos saudáveis, com qualidade e quantidades necessárias a fim de garantir a segurança alimentar e nutricional das famílias e favorecer a manutenção da agrobiodiversidade e os modos de vida tradicionais. É nesse contexto que a segurança alimentar e nutricional, ao considerar o respeito à diversidade étnica e cultural da população, torna-se um instrumento importante. Muito poderia ser refletido sobre isso, principalmente porque os quintais abrangem um espectro de plantas e animais e possui um caráter multifuncional. 


\section{Referências}

AMARAL, C. N.; GUARIM NETO, G. Os quintais como espaços de conservação e cultivo de alimentos: um estudo na cidade de Rosário Oeste (Mato Grosso, Brasil). Boletim Museu Paraense Emílio Goeldi: Ciências Humanas. Belém, v. 3, n. 3, 329-341, 2008.

CARNEIRO, M. J.; MALUF, R. S. (Orgs.). Para além da produção: multifuncionalidade e agricultura familiar. Rio de Janeiro: Mauad, 2003.

DIETARY REFERENCE INTAKES - DRIs. 2001. Disponível: <http://www.nap.edu.>. Acesso em: 15 fev. 2013.

EICHEMBERG, M. T.; AMOROZO, M. C. M.; MOURA, L. C. Species composition and plant use in old urban homegardens in Rio Claro, Southeast of Brazil. Acta Bot. Bras., São Paulo, v. 23, n. 4, 2009.

FOOD AND AGRICULTURE ORGANIZATION - FAO. O estado da segurança alimentar e nutricional no Brasil: um retrato multidimensional - Relatório 2014. Brasília, 2014. 90 p. Disponível em: <https://www.fao.org.br/download/SOFI_p.pdf>. Acesso em: 25 abr. 2014.

FREITAS, M. C. S.; PENA, P. G. L. Segurança alimentar e nutricional: a produção do conhecimento com ênfase nos aspectos da cultura. Rev. Nutr., Campinas, v. 20, n. 1, fev. 2007. Disponível em: < http://goo.gl/EIhYvC>. Acesso em: 13 maio 2014.

GALEAZZI, M. A. M. et al. Inquérito de consumo familiar de alimentos - Metodologia para identificação de famílias de risco alimentar. Revista Cadernos de Debate, Campinas, v. 4, p. 32-46, 1996.

GARBIN, V. H.; SILVA, M. J.; OLIVAL, A. Plano territorial de desenvolvimento rural sustentável território Baixada Cuiabana - MT. Brasília: MDA, 2006. 61 p. Disponível em: $<$ http://sit.mda.gov.br/download/ptdrs/ptdrs_territorio016.pdf>. Acesso em: 13 abr. 2014.

GARCIA Jr., A. R. O sul: caminho do roçado. Estratégias de reprodução camponesa e transformação social. São Paulo: Marco Zero; Brasília: Editora Universitária de Brasília; MCT CNPq, 1989.

INSTITUTO DE PESQUISA ECONÔMICA APLICADA - IPEA. A produção para autoconsumo no Brasil: uma análise a partir do censo agropecuário 2006. Relatório de pesquisa. Brasília, 2013.

KUMAR, B. Mohan.; NAIR, P. Ramachandran. The enigma of tropical homegardens. Agroforestry Systems, v. 61, n. 1-3, p. 135-152, 2004. 
MALUF, R. S. A multifuncionalidade da agricultura na realidade brasileira. In: MALUF, R.; CARNEIRO, M. J. (Orgs.). Para além da produção: multifuncionalidade e agricultura familiar. Rio de Janeiro: Mauad, 2003.

MATTOS, L.; BRONDIZIO, E.; ROMEIRO, A.; ORAIR, R. Agricultura de pequena escala e suas implicações na transição agroecológica na Amazônia Brasileira. Amazônica - Revista de Antropologia, v. 2, n. 2, p. 220-248, 2010.

MENASCHE, R.; MARQUES, F. C.; ZANETTI, C. Autoconsumo e segurança alimentar: a agricultura familiar a partir dos saberes e práticas da alimentação. Revista de Nutrição, Campinas, v. 21, p. 145-158, 2008.

MOREIRA, D. L.; GUARIM-NETO, G. Usos múltiplos de plantas do Cerrado: um estudo etnobotânico na comunidade sítio Pindura, Rosário Oeste, Mato Grosso, Brasil. Polibotánica, México, n. 27, abr. 2009.

NASCIMENTO, A. P. B. A migração como estratégia adaptativa em populações humanas rurais de Novo Cruzeiro, MG para Piracicaba, SP. 2003. 89 f. Dissertação (Mestrado em Ecologia de Agroecossistemas)- Escola Superior de Agricultura Luiz de Queiroz, Universidade de São Paulo, Piracicaba, 2003.

NIÑEZ, V. K. Household gardens: theorical considerations on a old survival strategy. Research series. Potatoes in food systems, v. 1, p. 1-41, 1984.

PASA, M. C. Etnobiologia de uma comunidade ribeirinha no alto da bacia do rio Aricá-Açú, Cuiabá, Mato Grosso, Brasil. 2004. 174 f. Tese (Doutorado em Ciências) - Centro de Ciências Biológicas e da Saúde, Universidade Federal de São Carlos, São Carlos, 2004.

PIERONI, A.; PRICE, L. Eating and healing. Traditional foods as medicine. New York: The Haworth Press, 2006.

RAMOS, M. O. A comida da roça ontem e hoje: um estudo etnográfico dos saberes e práticas alimentares dos agricultores de Maquiné (RS). 2007. 175 f. Dissertação (Mestrado em Desenvolvimento Rural) - Programa de Pós-graduação em Desenvolvimento Rural, Universidade Federal do Rio Grande do Sul, Porto Alegre, 2007.

SANTOS, S. Um estudo etnoecológico dos quintais de Alta Floresta - MT. 2004. 166 f. Dissertação (Mestrado em Ecologia e Conservação)- Programa de Pós-graduação em Ecologia e Conservação da biodiversidade, Universidade Federal de Mato Grosso, Cuiabá, 2004.

TABELA brasileira de composição de alimentos - TACO/NEPA. 4. ed. Campinas: Unicamp, 2011.

WEDIG, J. C. Agricultoras e agricultores à mesa: um estudo sobre campesinato e gênero a partir da antropologia da alimentação. 2009. 167 f. Dissertação (Mestrado em Desenvolvimento Rural)- 
Programa de Pós-graduação em Desenvolvimento Rural, Universidade Federal do Rio Grande do Sul, Porto Alegre, 2009.

ZANNETI, C.; MENASHE, R. Segurança alimentar, substantivo feminino: mulheres agricultoras e autoconsumo. In: MENASHE, R. A agricultura familiar à mesa: saberes e práticas da alimentação no Vale Taquari. Porto Alegre: Ed. UFRGS, 2007.

WOORTMANN, E. F. Padrões tradicionais e modernização: comida e trabalho entre camponeses teuto-brasileiros. In: MENASCHE, R. (Org.). A agricultura familiar à mesa: saberes e práticas da alimentação no Vale do Taquari. Porto Alegre: Ed. UFRGS, 2007.

Artigo recebido em 20/04/2016. Aceito para publicação em 14/06/2016. 